\title{
Fusion induced reversal of dendritic cell maturation: An altered expression of inflammatory chemokine and chemokine receptors in dendritomas
}

\author{
MELISSA BRANHAM-O'CONNOR ${ }^{1}$, JINHUA LI ${ }^{2}$, HARI S.R. KOTTURI ${ }^{1}$, \\ XIANZHONG YU ${ }^{1,2}$, THOMAS E. WAGNER ${ }^{1,2}$ and YANZHANG WEI ${ }^{1,2}$ \\ ${ }^{1}$ Department of Biological Sciences, Clemson University, Clemson; \\ ${ }^{2}$ Clemson University Biomedical Institute, Greenville, SC, USA
}

Received August 21, 2009; Accepted October 9, 2009

DOI: $10.3892 /$ or_00000668

\begin{abstract}
Dendritic cell-mediated cancer immunotherapy employs several ways to engage tumor antigens. We have demonstrated in both pre-clinical animal studies and early clinical trials that dendritomas, highly purified hybrids between dendritic cells (DC) and tumor cells, are superior activators of anti-tumor immunity. It has been argued, however, that DC vaccines may be dysfunctional in lymph node migration. In the present study we examined inflammatory chemokine and chemokine receptor expression as well as other maturation induced genes in dendritomas produced from either immature or mature DCs in order to shed light on their capacity to migrate from injection sites to draining lymph nodes and elicit an appropriate immune response. RNA microarray analysis was used to identify gene expression profiles for inflammatory chemokines and receptors and other maturation induced genes within dendritomas, lysate-pulsed dendritic cells, immature DCs and mature DCs. Gene regulation was confirmed with relative quantification, real-time RT-PCR in a separate experiment. We found that fusion of immature DCs to tumor cells initiates maturation with respect to inflammatory chemokines, chemokine receptors and other maturation induced genes in a similar pattern as LPS matured DCs. Interestingly, we saw a reversed gene profile when mature DCs were fused to tumor cells. LPS matured DCs displayed the chemokine repertoire expected with DC maturation; however, once fused to tumor cells, these chemokines and other maturation induced genes reverted to levels comparable to immature DCs. It appears that mature DCs used for dendritoma production result in a de-mature genotype. Our results indicate that dendritomas from immature DC/tumor
\end{abstract}

Correspondence to: Dr Yanzhang Wei, Clemson University Biomedical Institute, Greenville, SC 29605, USA

E-mail: ywei@ghs.org

Key words: dendritoma, dendritic cell fusion, chemokines, microarray, real-time PCR cell fusions may be more effective in migration from injection site to draining lymph nodes and, therefore, would be more effective in stimulating anti-tumor immunity.

\section{Introduction}

Dendritic cells (DC) are professional antigen presenting cells, which play a vital role in stimulating immune responses against infections and tumor cells (1-3). DC-mediated cancer immunotherapy is aimed at picking up where the host immune system failed by presenting tumor antigens to innate and adaptive effector cells, thus stimulating anti-tumor immunity for immediate therapy and latent protection (4-6). Three basic approaches have been employed to engage DCs with tumor antigens: tumor antigen pulsing, genetic modification with tumor antigen genes or RNA, and DC/tumor fusion $(7,8)$. Although all three approaches have been widely utilized and have successfully increased tumor-antigen reactive $T$ cells in periphery, the $\mathrm{DC} /$ tumor hybridoma vaccine has proved more effective since this strategy provides a broader diversity of known and unknown tumor antigens as well as MHC class I and MHC class II antigens to the immune system $(9,10)$.

Most DC hybridoma studies have utilized fusion mixtures as a vaccine due to the lack of selective markers on fused DC/tumor cells to purify hybrids from the fusion mixture (11). The immune response stimulated by this mixture is compromised due to the presence of large numbers of unfused cells or self/self fused cells. In order to solve this problem, we developed a novel hybrid purification technology that instantly purifies DC/tumor hybrids from the mixture (12). Animal studies demonstrated that highly purified DC/ tumor hybrids, or dendritomas (DT), are superior activators to stimulate anti-tumor immunity compared with fusion mixtures (13). Several clinical trials using dendritoma vaccines have been conducted; and data show that DT vaccines stimulate anti-tumor immune responses in some patients and demonstrate observable clinical responses $(14,15)$.

Although most DC hybridoma vaccines were effective in preclinical animal studies, clinical trials have shown less encouraging results (16). Consequently, the hottest field in DC-mediated cancer immunotherapy is to understand and 
solve the inconsistencies between animal studies and human clinical trials. The increase of regulatory $\mathrm{T}$ cells and tolerogenic DCs found in tumors after DC vaccine administration are two of the major factors suppressing antitumor immunity (17-19). Others include DC procurement, route of administration, and tumor microenvironment (20-22). The overall belief is that DCs must be presented with maturation stimuli and tumor antigen, administered through an appropriate route for different cancers with mediators aiding their lymph node migration, and have the capacity to process and present both MHC I and II peptides in order to acquire therapeutic and long-term protective immunity (23). Much research has been done to progress DC vaccination in most of these areas; however information on the migratory capacity of DC vaccines still needs attention. It is imperative to understand the factors involved in the migration of dendritomas, and other DC vaccines, to the draining lymph nodes where they encounter and activate effector $\mathrm{T}$ cells.

Inflammatory chemokines, such as macrophage inflammatory protein $1 \alpha(\mathrm{MIP}-1 \alpha)$ and RANTES (CCL3 and CCL5, respectively), are predominantly located at non-lymphatic sites of inflammation where they recruit immune cells to participate in antigen presentation and recognition to ultimately elicit a cell-mediated response to infection or tumor cells. Immature DCs (iDC) typically express inflammatory chemokine receptors CCR1, CCR2 and CCR5 which bring them into contact with antigens at inflammatory sites (24). Once antigen uptake has ensued, DCs rapidly increase production of inflammatory chemokines and lose responsiveness to these CCLs, a process called autodesensitization (25), allowing for reverse transmigration of activated, mature DCs (mDC) into secondary lymphoid tissues where they present antigen to effector cells. Clearly, the completion of this process is essential for effective DC vaccines. In the present study, in order to understand whether dendritomas are capable of effective migration to secondary lymphoid tissues, we examined the regulation of key chemokines and chemokine receptors along with several maturation induced genes. Our microarray and real-time RT-PCR results demonstrate that fusing tumor cells to iDCs matures them with respect to $c c r$ and $c c l$ expression, while fusing tumor cells to mDCs causes the reversal of $c c r$ and $c c l$ expression by mDCs. Our results implicate immature DCs as better choice for dendritoma production, and a migratory mediator adjuvant may be needed when mature DCs are used for dendritoma production.

\section{Materials and methods}

Mice and tumor cells. Female C57BL/6J mice at 6-8 weeks of age were purchased from Jackson Laboratories (Bar Harbor, ME) and housed in our pathogen-free animal facilities. Animal experiments were carried out in accordance with both Guidelines for the Care and Use of Laboratory Animals (NIH publication no. 85-23) and institutional guidelines. Murine acute myeloid leukemia cell line C1498 and murine melanoma cell line B16F0, both C57BL/6J-derived, were maintained in complete DMEM (Gibco BRL, Grand Island, NY) supplemented with 10\% FBS (Hyclone, Logan, UT) and $50 \mu \mathrm{g} / \mathrm{ml}$ gentamicin (Gibco BRL) at $37^{\circ} \mathrm{C}$ in a humidified atmosphere of $5 \% \mathrm{CO}_{2}$.
Dendritic cells. Bone marrow derived DCs were cultured as previously described (26). Briefly, bone marrow cells flushed from C57BL/6J mouse femurs and tibiae with RPMI-1640 (Gibco BRL) were filtered through $40-\mu$ m nylon cell strainers. After the removal of RBCs by ACK lysate (Lonza, Allendale, $\mathrm{NJ}$ ), the remaining cells were resuspended in DC medium containing RPMI-1640 supplemented with 10\% FBS, $50 \mu \mathrm{g} /$ $\mathrm{ml}$ gentamicin and $20 \mathrm{ng} / \mathrm{ml} \mathrm{rmGM-CSF}$ (Sigma, St. Louis, MO) and plated at $4-5 \times 10^{6}$ cells $/ 10 \mathrm{ml}$ in a $100-\mathrm{mm}$ tissue culture dish. On day 4, $10 \mathrm{ml}$ fresh DC media was added to each dish. On day 8, non-adherent and loosely adherent cells were harvested, washed with RPMI-1640 and replated in fresh DC medium containing $10 \mathrm{ng} / \mathrm{ml} \mathrm{rmGM-CSF}$ with or without $100 \mathrm{ng} / \mathrm{ml}$ LPS (Sigma). On day 10, nonadherent and loosely adherent cells were collected for further studies.

Pulsing DCs with tumor lysate. B16F0 cells were collected and resuspended in a conical tube in $1 \mathrm{X}$ PBS at a concentration of $1 \times 10^{7}$ cells $/ \mathrm{ml}$. The tube with cell suspension was immersed in a dry-ice/methanol bath for $\sim 3 \mathrm{~min}$. Once frozen, the cells were placed in a $37^{\circ} \mathrm{C}$ water bath with gentle agitation and thawed completely. The process was repeated for a total of four freeze/thaw cycles. The cells were then centrifuged at $15000 \mathrm{x}$ g for $10 \mathrm{~min}$ at $20^{\circ} \mathrm{C}$ and supernatant was collected. Protein concentration was determined using the Bio-Rad Protein Assay (Bio-Rad, Hercules, CA). Either immature DCs (iDC) or LPS matured DCs (mDC) were incubated with $100 \mu \mathrm{g} / \mathrm{ml}$ tumor protein lysate overnight. Pulsed DCs (LPiDC or LPmDC) were then centrifuged at $300 \mathrm{x}$ g to collect cells but discard lysate in the supernatant. Cells were washed three times in $1 \mathrm{X}$ PBS prior to RNA extraction.

Cell staining and fusion. DCs and tumor cells were stained green and red, respectively, using PKH67-GL or PKH26-GL kits (Sigma) according to manufacturer's protocol. Stained cells were washed thrice to remove unbound dye and tumor cells were irradiated with $50 \mathrm{~Gy}$. Tumor cells and DCs were fused at a ratio of $1: 1$ or 1:2 using a 50\% PEG $10 \%$ DMSO solution (Sigma). After fusion, cells were incubated overnight in DC medium.

FACS sorting. The fusion mixtures were harvested (both adherent and non-adherent) and resuspended in PBS at a concentration of $1 \times 10^{7}$ cells $/ \mathrm{ml}$. Cells were sorted on a BD FACSCalibur (Becton-Dickinson, San Jose, CA) according to the dual fluorescent colors. Sorted cells, labelled as dendritomas (DT), were resuspended in DC medium and incubated overnight with or without $100 \mathrm{ng} / \mathrm{ml}$ LPS prior to RNA extraction. Dendritomas exhibited both green and red fluore-scence and purity was $>95 \%$.

Microarray. LPiDC, LPmDC, DT, DC and tumor cell RNA was extracted using ArrayGrade ${ }^{\mathrm{TM}}$ Total RNA Isolation kit (SABiosciences, Frederick, MD) and was sent for pathwayfocused GEArray service using mouse Dendritic and Antigen Presenting Cell Oligo GEArray (SABiosciences). Analysis was performed using the GEArray Expression Analysis Suite software (SABiosciences). 


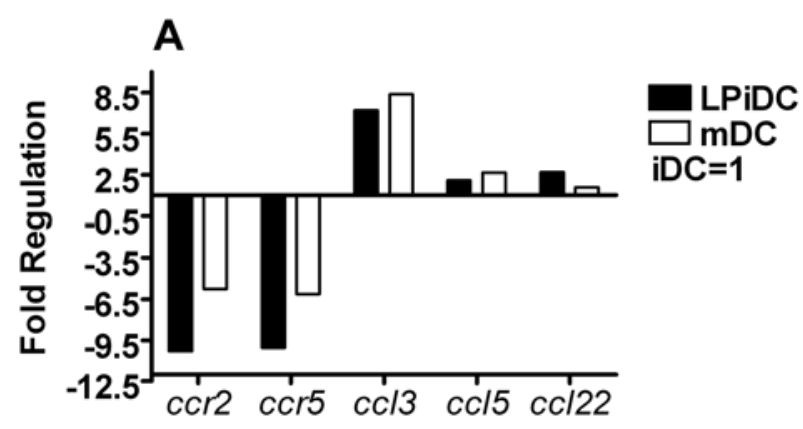

B

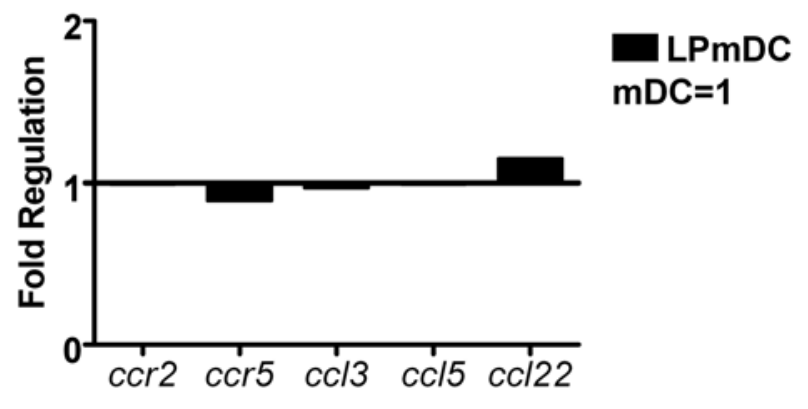

Figure 1. Tumor lysate pulsing matures iDC, but causes no change in $\mathrm{mDC}$. Total RNA was extracted from iDC, LPiDC, $\mathrm{mDC}$ and LPmDC and was analyzed by RNA microarray for the indicated chemokine and chemokine receptors. (A) Gene expression profiles of LPiDC and $\mathrm{mDC}$ compared to iDC. (B) Gene expression profiles of LPmDCs compared to mDC.

Real-time PCR. Real-time one-step RT-PCR was performed on total RNA via an Eppendorf Mastercycler ep Realplex ${ }^{2}$ (Eppendorf, Westbury, NY) using QuantiTect Primers optimized for QuantiTect SYBR Green RT-PCR kit (Qiagen, Valencia, CA). Results were normalized to $B$-actin, which was chosen over GAPDH and B2M as the housekeeping gene, since it was least affected by treatment. Data are analyzed by $\Delta \Delta \mathrm{Ct}$ calculations.

\section{Results}

Tumor lysate matures iDCs. Overnight incubation of iDC with B16F0 tumor cell lysate induced DC maturation with respect to inflammatory chemokine and chemokine receptors. iDCs express high levels of inflammatory receptors: CCR2 and CCR5 and low levels of inflammatory chemokines: CCL3, CCL5 and CCL22. Upon antigen uptake and processing, iDCs are induced to decrease levels of inflammatory receptors, while increasing inflammatory chemokine expression (27). Microarray analysis shows tumor lysate pulsed iDCs (LPiDCs) displayed a drastic reduction in $c c r 2$ and $c c r 5$ and an increase in inflammatory and inducible chemokines $c c l 3, c c l 5$ and $c c l 22$; their levels were nearly identical to DCs matured with LPS (Fig. 1A).

Tumor lysate has no effect on mDCs. DCs cultured from C57BL/6J mice were matured with LPS on day 8 of culture. On day 10, they were incubated with B16F0 tumor lysate and RNA was extracted after overnight incubation. RNA micro-
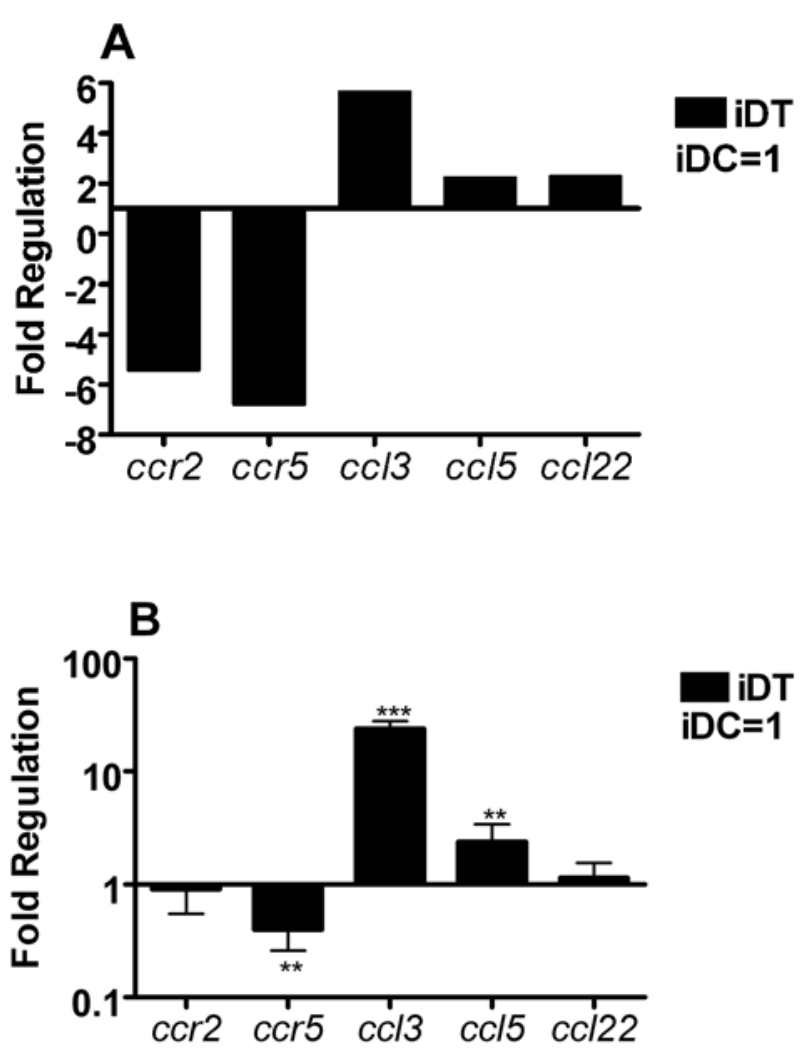

Figure 2. Fusion with tumor cells matures iDC. iDCs were fused with C1498 or B16F0 tumor cells in a $1: 1$ or $2: 1$ ratio using PEG in two separate experiments. The fusion hybrids (iDT) were generated by FACS sorting based on the DT technology (Materials and methods). Total RNA was analyzed by RNA microarray and real-time RT-PCR for the indicated genes. (A) Microarray analysis of chemokine and chemokine receptor gene expression by iDTs, iDC=1. (B) Real-time RT-PCR analysis of chemokine and chemokine receptor gene expression by iDTs, iDC $=1 .\left({ }^{*} \mathrm{p}<0.05,{ }^{* *} \mathrm{p}<0.01\right.$, *** $\mathrm{p}<0.001)$.

array shows that tumor lysate pulsing of $\mathrm{mDCs}(\mathrm{LPmDC})$ caused no change in expression of $c c r 2, c c r 5, c c l 3, c c l 5$ or ccl22 compared to LPS matured DCs (mDC, Fig. 1B).

Fusion with tumor cells matures iDC. iDCs were fused with B16F0 tumor cells by PEG in a 2:1 ratio. The fused hybrids (immature dendritomas or iDT) were purified from the fusion mixture by dual fluorescent FACS sorting on day 11. RNA was extracted from iDTs following collection and used for RNA microarray. As shown in Fig. 2A, iDTs dramatically decreased expression of $c c r 2$ and $c c r 5$, but increased $c c l 3$, $c c l 5$ and $c c l 22$ as compared to iDCs. This pattern is consistent with the expression of mDCs and LPiDCs (Fig. 1A); therefore, fusion of iDC with tumor cells instigates maturation with respect to these inflammatory chemokines and receptors. To further confirm this finding, real-time RT-PCR was performed to measure the change of expression in $c c r 2, c c r 5$, $c c l 3, c c l 5$ and $c c l 22$ in iDTs. The results, as shown in Fig. 2B, demonstrate a similar pattern of expression: down-regulation of $c c r$ genes and up-regulation of $c c l$ genes.

Additional genes associated with LPS induced DC maturation were examined in two independent microarray experiments in iDTs made from either C1498 tumor cells or 

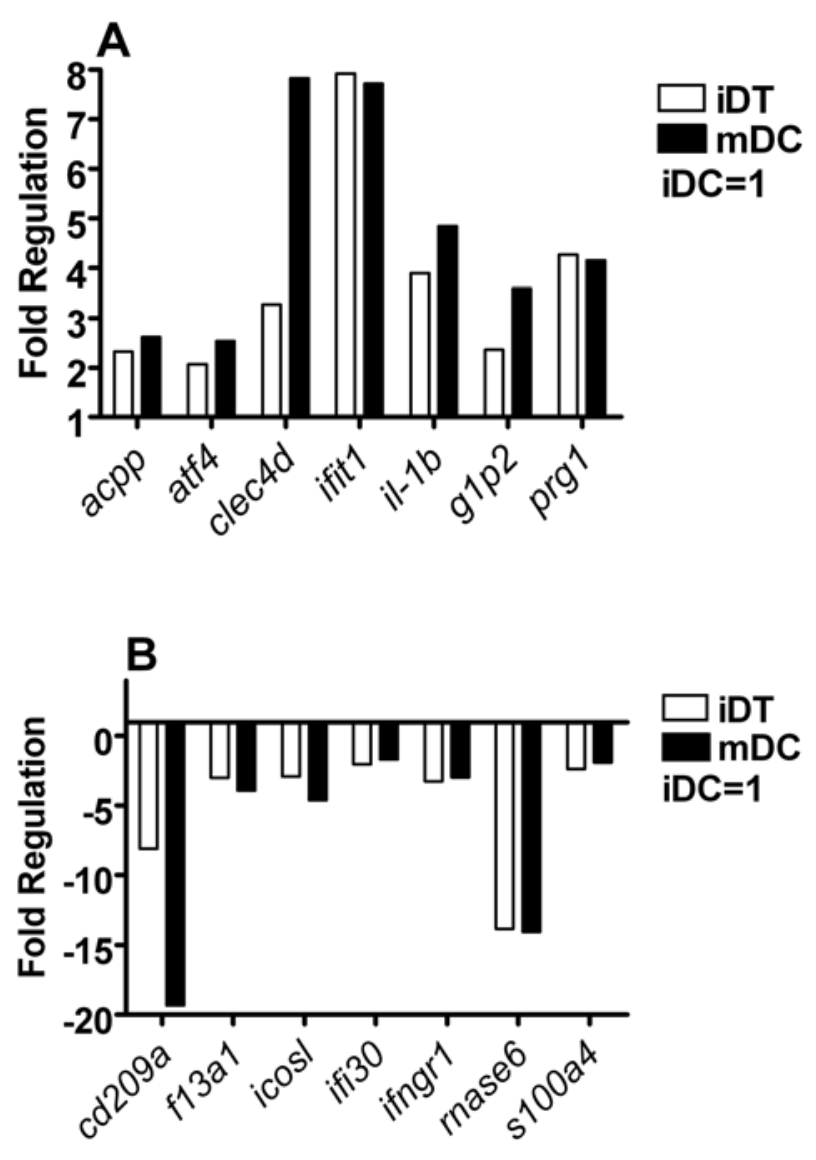

Figure 3. Fusion of iDCs with tumor cells yields a similar gene profile to LPS matured DCs. iDCs were fused with C1498 or B16F0 tumor cells in a 1:1 or 2:1 ratio using PEG in two separate experiments. The fusion hybrids (iDT) were generated by FACS sorting based on the DT technology (Materials and methods). Total RNA was analyzed by RNA microarray for the indicated genes. (A) Genes up-regulated by both iDTs and LPS matured DCs compared to iDCs. (B) Genes down-regulated by iDTs and LPS matured DCs compared to iDCs.

B16F0 tumor cells. As shown in Fig. 3, acpp, atf4, clec4d, ifit1, il-1b, g1p2 and prg1 were up-regulated (Fig. 3A), while cd209a, f13al, icosl, ifi30, ifngrl, rnase6 and s100a4 were down-regulated (Fig. 3B), indicating that fusion of iDCs and tumor cells yields a maturing hybridoma.

Fusion with tumor cells reverses the maturation for mDTs. DCs cultured for 8 days were matured with LPS for two days and fused with B16F0 tumor cells. The hybrids (mature dendritomas or $\mathrm{mDT}$ ) were purified from the fusion mixture using the same technology described above. RNA microarray established the increased expression of $c c r 2$ and $c c r 5$, while the expression of $c c l 3, c c l 5$ and $c c l 22$ decreased (Fig. 4A): a clear pattern of DC de-maturation with respect to inflammatory $c c l$ and $c c r$. This finding is in contrast to lysate pulsed mDCs where no significant change was observed (Fig. 1B). Real-time RT-PCR also confirmed the change of expression in $c c r 2, c c r 5, c c l 3, c c l 5$ and $c c l 22$ in mDTs. The results, shown in Fig. 4B, are consistent with microarray data: $c c r$ genes are up-regulated and $c c l$ genes down-regulated. Additional genes of interest analyzed by microarray of mDTs showed a similar pattern compared to iDCs. cd209a, fl3al,
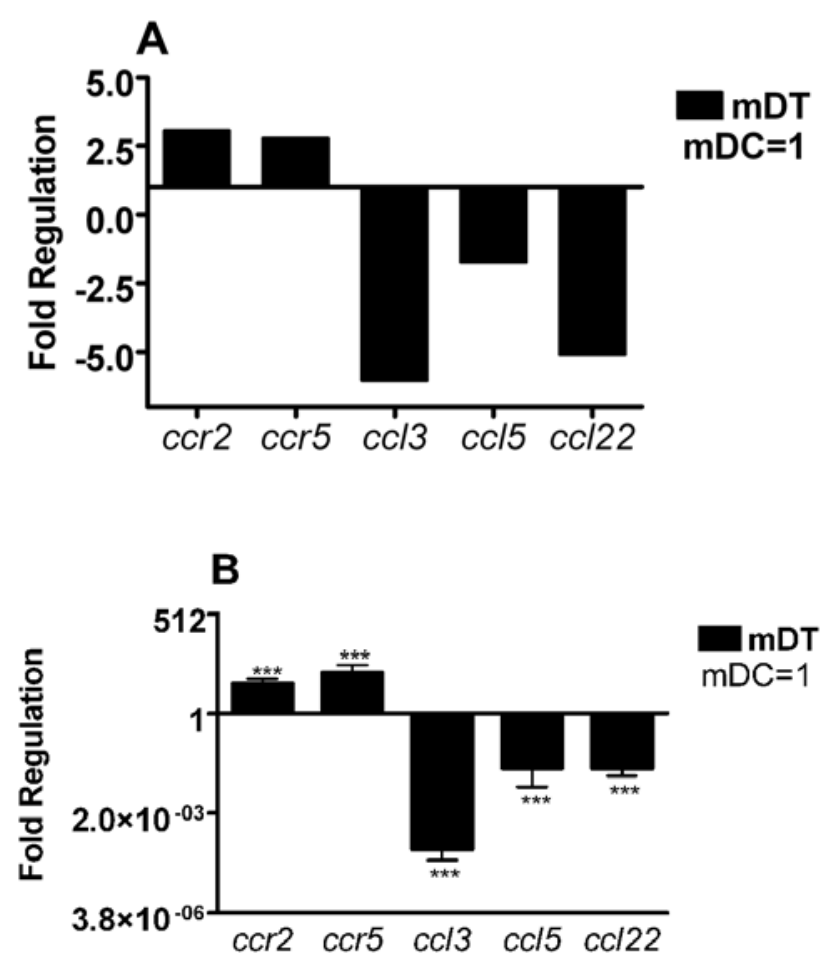

Figure 4. Fusion of mDCs with tumor cells (mDT) yields an opposing chemokine/chemokine receptor pattern to mature DCs. mDCs were fused with B16F0 tumor cells in a 2:1 ratio using PEG. The fusion hybrids, mDT, were generated by FACS sorting based on the DT technology (Materials and methods). Total RNA was analyzed by RNA microarray and real-time RTPCR for the indicated genes. (A) Microarray analysis of chemokine and chemokine receptor gene expression by $m D T s, m D C=1$. (B) Real-time RT-PCR analysis of chemokine and chemokine receptor gene expression by mDTs, $\mathrm{mDC}=1 .\left({ }^{*} \mathrm{p}<0.05,{ }^{* *} \mathrm{p}<0.01,{ }^{* * *} \mathrm{p}<0.001\right)$.

marcks, rpl13a, cd207, cdc42 and pfn1 were up-regulated (Fig. 5A), while acpp, atf4, btg1, clec4d, il-1b, il-12b, cd36, sod2, cd80, pnrc1 and tnfsf4 were down-regulated (Fig. 5B), further supporting de-maturation of mDTs.

\section{Discussion}

Tumor lysate pulsed immature DCs (LPiDCs) are mature compared to iDCs. LPiDCs express increased levels of CD80, CD86 and CD40 costimulatory molecules as well as molecules involved in antigen presentation while decreasing those involved with antigen uptake $(28,29)$. For the first time, we show that LPiDCs are activated to elevate $c c l$ levels and decrease ccr levels (Fig. 1A), suggesting that antigen-pulsing results in efficient DC activation to mediate the chemokine receptor paradigm switch in the absence of other inflammatory stimuli or microbial products such as LPS.

We did not observe any significant change in the genes analyzed by microarray for tumor lysate pulsed, LPS matured DCs (LPmDCs, Fig. 1B). This could be due to the notion that LPS matured DCs have down-regulated antigen-uptake mechanisms, or compared to microbial danger signals, tumor lysates are weak DC maturation agents. Interestingly, it has been shown that coincubating mDCs with CCR7 ligands, CCL19/ELC or CCL21/SLC, re-stimulates endocytosis by 

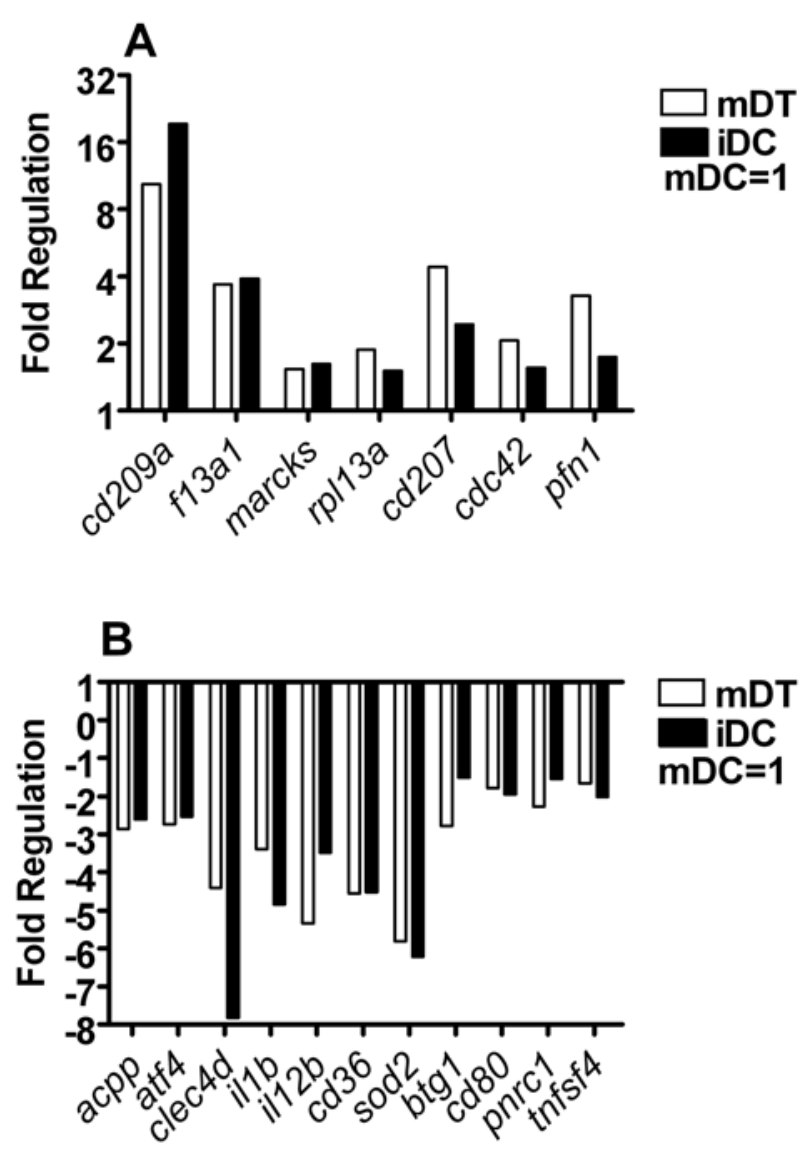

Figure 5. Fusion of mDCs with tumor cells de-matures mDTs. mDCs were fused with B16F0 tumor cells in a 2:1 ratio using PEG. The fusion hybrids, mDT, were generated by FACS sorting based on the DT technology (Materials and methods). Total RNA was analyzed by RNA microarray for the indicated genes. (A) Genes up-regulated by both mDTs and iDCs compared to mDCs. (B) Genes down-regulated by mDTs and iDCs compared to mDCs.

previously matured DCs (30); therefore, it seems plausible to induce mDC uptake of tumor lysate by concomitant incubation with a CCR7 ligand. Based on our data, it would be better to use iDCs to engage tumor antigens; nonetheless, it would be interesting to observe the effects of pulsing mDCs with tumor lysate combined with ELC or SLC.

iDTs, composed of tumor cells and iDCs, express increased levels of $c c l$ genes and decreased $c c r$ genes (Fig. 2). This process is assumed to be mostly due to the autocrine action of chemokines on their respective receptors expressed by DCs $(33,34)$; although it has been shown that C5a and fMPL do not have this autodesensitization effect on DCs (24). In addition to the $c c l$ and $c c r$ paradigm switch, iDT maturation is also accompanied with up-regulation of genes such as acpp, atf4, clec4d, ifit1, il-1b, g1p2 and prg1 and downregulation of $c d 209 a, f 13 a 1$, icosl, ifi30, ifngrl, rnase6 and s100a4 as confirmed in two separate microarray analyses and also observed in canonical DC maturation when iDCs are matured with LPS (Fig. 3). Again, our data points to successful maturation when iDCs are fused to tumor cells (iDTs) which is important when designing DC vaccines. Interestingly, we observed that iDTs expressed high levels of il-10 in two separate microarrays, while mDTs expressed non-detectable levels of $i l-10$ (data not shown), contradicting other reports where both iDC/tumor fusions and $\mathrm{mDC} /$ tumor fusions have elevated levels of IL-10 (35). This may indicate the need for simultaneous addition of a danger signal to overcome IL-10 production when manufacturing iDTs. More studies are needed to clarify the role of IL-10 in this process.

Mature DCs used for fusion oddly de-mature, whereas iDCs are matured with fusion to tumor cells. Interestingly, the $c c l$ and $c c r$ expression of mDTs was completely reversed compared to iDTs and mDCs. There was a severe decrease in $c c l$ expression with a compensating increase in $c c r$ expression (Fig. 4), closely mimicking the $c c r$ and $c c l$ expression of iDCs. This phenomenon may be due to complete washing of mDCs before fusion, which frees the supernatant of CCLs, no longer triggering autodesensitization. Taking this into account, mDTs may not be as good as iDTs for migration from injection sites to draining lymph nodes. Not only did mDTs de-mature with respect to $c c r$ and $c c l$ expression, but they also up-regulated $c d 209 a, f 13 a 1$, marcks, rpl13a, cd207, $c d c 42$ and $p f n 1$, and down-regulated acpp, atf4, btg1, clec4d, il-1b, il-12b, cd36, sod2, cd80, pnrcl and tnfsf4 (Fig. 5) in syn-chrony with iDCs. Our data suggest mDTs may need restimu-lation with CCLs or possibly other danger signals in order to resume proper migratory capacity and antigen presentation since they seem to have more genes in common with iDCs than mDCs. It is possible that stimulating dendritomas with microbial danger signals or adjuvants could overcome this problem as seen with pre-treatment and posttreatment with OK432 and $\mathrm{CpG}$ ODN increasing the effectiveness of DC fusion vaccines $(31,36)$.

In conclusion, our data unfold a significant pattern of chemokine expression in dendritomas depending on the maturation state of DCs and demonstrates the plasticity of LPS matured DCs used for vaccine preparation. Although mature DCs are preferred to reduce tolerance or expansion of regulatory cells, we see that there may be problems with migration and induction of immune response. To circumvent this problem, either immature DCs should be used for fusion with simultaneous addition of a danger signal such as LPS, OK432 or CpG ODN to properly mature the DCs and abrogate production of IL-10; or if mature DCs are used for fusion, it must be investigated to see if they can be re-stimulated to secrete CCLs, down-regulate CCRs, and reclaim maturation in order to properly migrate and activate an immune response against presented tumor antigens.

\section{Acknowledgements}

We would like to thank Eric Holle and his staff for the professional care of mice used in this study, Xinhai Zhang for critical review and discussion of data, Leigh Theofanous and Hilary Bouton-Verville for acquisition of data, and Keri Nowend for help with statistical design. This study is partially supported by the GHS Oncology Endowment and the William K. and Frances J. Bryan New Hope Fund for Cancer Research.

\section{References}

1. Banchereau J and Steinman RM: Dendritic cells and the control of immunity. Nature 392: 245-252, 1998.

2. Hart DN: Dendritic cells: unique leukocyte populations which control the primary immune response. Blood 90: 3245-3287, 1997. 
3. Dunn GP, Bruce AT, Ikeda H, Old LJ and Schreiber RD: Cancer immunoediting: from immunosurveillance to tumor escape. Nat Immunol 3: 991-998, 2002.

4. Ullrich E, Menard C, Flament C, Terme M, Mignot G, Bonmort M, Plumas J, Chaperot L, Chaput N and Zitvogel L: Dendritic cells and innate defense against tumor cells. Cytokine Growth Factor Rev 19: 79-92, 2008.

5. Banchereau J and Steinman RM: Exploiting dendritic cells to improve vaccine efficacy. J Clin Invest 109: 1519-1526, 2002.

6. Steinman RM and Hemmi H: Dendritic cells: translating innate to adaptive immunity. Curr Top Microbiol Immunol 311: 17-58, 2006.

7. Schuler G, Schuler-Thurner B and Steinman RM: The use of dendritic cells in cancer immunotherapy. Curr Opin Immunol 15: 138-147, 2003

8. Svane IM, Soot ML, Buus S and Johnsen HE: Clinical application of dendritic cells in cancer vaccination therapy. APMIS 111: 818-834, 2003

9. Ward S, Casey D, Labarthe MC, Whelan M, Dalgleish A, Pandha $\mathrm{H}$ and Todryk S: Immunotherapeutic potential of whole tumor cells. Cancer Immunol Immunother 51: 351-357, 2002.

10. Shimizu K, Kuriyama H, Kjaergaard J, Lee W, Tanaka H and Shu S: Comparative analysis of antigen loading strategies of dendritic cells for tumor immunotherapy. J Immunother 27: 265-272, 2004

11. Haigh P, Difronzo L, Gammon G and Morton DL: Vaccine therapy for patients with melanoma. Oncology 13: 1561-1574, 1999.

12. Holmes LM, Li J, Sticca RP, Wagner TE and Wei Y: A rapid, novel strategy to induce tumor cell-specific cytotoxic T lymphocyte responses using instant dendritomas. J Immunother 24: $122-129,2001$

13. Li J, Holmes LM, Franek KJ, Burgin KE, Wagner TE and Wei Y: Purified hybrid cells from dendritic cell and tumor cell fusions are superior activators of antitumor immunity. Cancer Immunol Immunother 50: 456-462, 2001.

14. Wei Y, Sticca RP, Holmes LM, Burgin KE, Li J, Williamson J, Evans L, Smith SJ, Stephenson JJ and Wagner TE: Dendritoma vaccination combined with low dose interleukin-2 in metastatic melanoma patients induced immunological and clinical responses. Int J Oncol 28: 585-593, 2006.

15. Wei YC, Sticca RP, Li J, Holmes LM, Burgin KE, Jakubchak S, Bouton-Verville H, Williamson J, Meyer K, Evans L, Martin J, Stephenson JJ, Trocha S, Smith S and Wagner TE: Combined treatment of dendritoma vaccine and low-dose interleukin-2 in stage IV renal cell carcinoma patients induced clinical response: a pilot study. Oncol Rep 18: 665-671, 2007.

16. Gong JL, Koida S and Calderwood SK: Cell fusion: from hybridoma to dendritic cell-based vaccine. Expert Rev Vaccines 7: $1055-1068,2008$

17. Steinman RM, Hawiger D and Nussenzweig MC: Tolerogenic dendritic cells. Annu Rev Immunol 21: 685-711, 2003.

18. Li J, Theofanous L, Stickel S, Bouton-Verville H, Burgin KE, Jakubchak S, Wagner TE and Wei Y: Transfer of in vitro expanded $\mathrm{T}$ lymphocytes after activation with dendritomas prolonged survival of mice challenged with EL4 tumor cells. Int J Oncol 31: 193-197, 2007.

19. Dannull J, Su Z, Rizzieri D, Yang BK, Coleman D, Yancey D, Zhang AJ, Dahm P, Chao N, Gilboa E and Vieweg J: Enhancement of vaccine-mediated antitumor immunity in cancer patients after depletion of regulatory T cells. J Clin Invest 115: 3623-3633, 2005

20. Melief CJ: Cancer immunotherapy by dendritic cells. Immunity 29: 372-383, 2008

21. Grover A, Kim GJ, Lizée G, Tschoi M, Wang G, Wunderlich JR, Rosenberg SA, Hwang ST and Hwu P: Intralymphatic dendritic cell vaccination induces tumor antigen-specific, skin-homing $\mathrm{T}$ lymphocytes. Clin Cancer Res 12: 5801-5808, 2006.
22. Fricke I and Gabrilovich DI: Dendritic cells and tumor microenvironment: a dangerous liaison. Immunol Invest 35: 459-483, 2006.

23. Ueno H, Fay J, Banchereau J and Palucka AK: Dendritic cell subsets as targets and vectors for vaccination: dendritic cells as cancer vaccines. In: Handbook of Dendritic Cells: Biology, Diseases and Therapies. Lutz MB, Romani N and Steinkasserer A (eds). Wiley-VCH Verlag GmbH \& Co. KGaA, Weinheim, pp1095-1115, 2006.

24. Sozzani S, Allavena P, Vecchi A and Mantovani A: Chemokines and dendritic cell traffic. J Clin Immunol 20: 151-160, 2000.

25. Sallusto F and Lanzavecchia A: Understanding dendritic cell and T-lymphocyte traffic through the analysis of chemokine receptor expression. Immunol Rev 177: 134-140, 2000.

26. Lutz MB, Kukutsch N, Ogilvie ALJ, Robner S, Koch R, Romani N and Shuler G: An advanced culture method for generating large quantities of highly pure dendritic cells from mouse bone marrow. J Immunol Methods 223: 77-92, 1999.

27. Sallusto F, Schaerli P, Loetscher P, Schaniel C, Lenig D, Mackay CR, Qin S and Lanzavecchia A: Rapid and coordinated switch in chemokine receptor expression during dendritic cell maturation. Eur J Immunol 28: 2760-2769, 1998.

28. Paglia P, Chiodoni C, Rodolfo M and Colombo MP: Murine dendritic cells loaded in vitro with soluble protein prime cytotoxic $\mathrm{T}$ lymphocytes against tumor antigen in vivo. J Exp Med 183: 317-322, 1996.

29. Bernard F, Blanco P, Davoust J, Neidhart-Berard EM, NouriShirazi M, Taquet N, Rimoldi D, Cerottini JC, Banchereau J and Palucka AK: Cross-priming of naïve CD8 T cells against melanoma antigens using dendritic cells loaded with killed allogeneic melanoma cells. J Exp Med 192: 1535-1543, 2000

30. Yanagawa Y and Onoe K: CCR7 ligands induce rapid endocytosis in mature dendritic cells with concomitant up-regulation of Cdc42 and Rac activities. Blood 101: 4923-4929, 2003

31. Li YL, Wu YG, Wang YQ, Li Z, Wang RC, Wang L and Zhang YY: Bone marrow-derived dendritic cells pulsed with tumor lysates induce anti-tumor immunity against gastric cancer ex vivo. World J Gastroenterol 14: 7127-7132, 2008.

32. Koido S, Hara E, Homma S, Torii A, Mitsunaga M, Yanagisawa S, Toyama Y, Kawahara H, Watanabe M, Yoshida S, Kobayashi S, Yanaga K, Fujise K and Tajiri H: Streptococcal preparation OK-432 promotes fusion efficiency and enhances induction of antigen-specific CTL by fusions of dendritic cells and colorectal cancer cells. J Immunol 178: 613-622, 2007.

33. Kikuchi T, Akasaki Y, Irie M, Homma S, Abe T and Ohno T: Results of a phase I clinical trial of vaccination of glioma patients with fusions of dendritic and glioma cells. Cancer Immunol Immunother 50: 337-344, 2001

34. Sozzani S, Allavena P, D'Amico G, Luini W, Bianchi G, Kataura M, Imai T, Yoshie O, Bonecchi R and Mantovani A: Cutting edge: differential regulation of chemokine receptors during dendritic cell maturation: a model for their trafficking properties. J Immunol 161: 1083-1086, 1998.

35. Vasir B, Wu Z, Crawford K, Rosenblatt J, Zarwan C, Bisssonnette A, Kufe D and Avigan D: Fusions of dendritic cells with breast carcinoma stimulate the expansion of regulatory $\mathrm{T}$ cells while concomitant exposure to IL-12, CpG oligodoexynucleotides, and anti-CD3/CD28 promote the expansion of activated tumor reactive T cells. J Immunol 181: 808-821, 2008.

36. Koido S, Hara E, Homma S, Mitsunaga M, Takahara A, Nagasaki E, Kawahara H, Watanabe M, Toyama Y, Yanagisawa S, Kobayashi S, Yanaga K, Fujise K, Gong J and Tajiri H: Synergistic induction of antigen-specific CTL by fusions of TLR-stimulated dendritic cells and heat-stressed tumor cells. J Immunol 179: 4874-4883, 2007. 